\title{
Multidisciplinary simulation of primary tracheal anastomosis and cross-field ventilation - scenario development and feasibility assessment
}

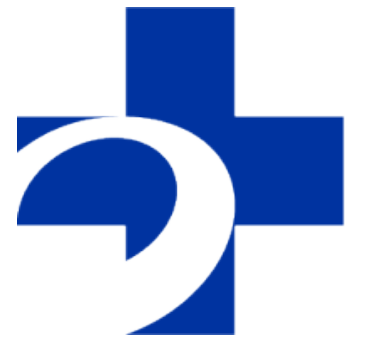

The Ottawa Hospital

RESEARCH INSTITUTE

Inspired by research. Inspiré par la recherche. Driven by compassion. Guidé par la compassion.

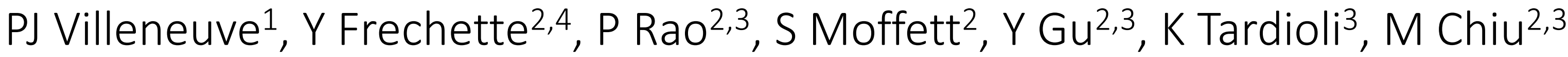

Division of Thoracic Surgery, Department of Surgery ${ }^{1}$, Department of Anaesthesiology and Pain Medicine ${ }^{2}$, uOttawa Skills and Simulation Centre ${ }^{3}$; The Ottawa Hospital, I'Université de Sherbrooke ${ }^{4}$ and The University of Ottawa

\section{Problem}

Tracheal resection and primary anastomosis is a relatively uncommon procedure. The surgical and anesthetic management is technically demanding, requiring a high level of collaboration and communication between the surgical and anesthesiology teams.

\section{Objective}

To develop and pilot a team-based, high-fidelity simulation to teach and maintain the necessary technical and non-technical skills needed to successfully perform a tracheal anastomosis.

\section{Methods}

The scenario was developed by experts from thoracic surgery, thoracic anaesthesia, and medical education/simulation. We developed a scenario that incorporated a technically challenging surgical task with the complex airway management decision-making. Tasks and objectives for learners were linked to Royal College objectives of training and CanMEDS roles (Medical Expert, Collaborator, Professional and Communicator). The physical model consisted of a transected porcine trachea, draped as a surgical field, laid on top of the Laerdal SimMan. Learners filled out pre-and-post scenario questionnaires scored on a 5-point Likert scale, related to the familiarity of the techniques of tracheal anastomosis and cross-field ventilation.

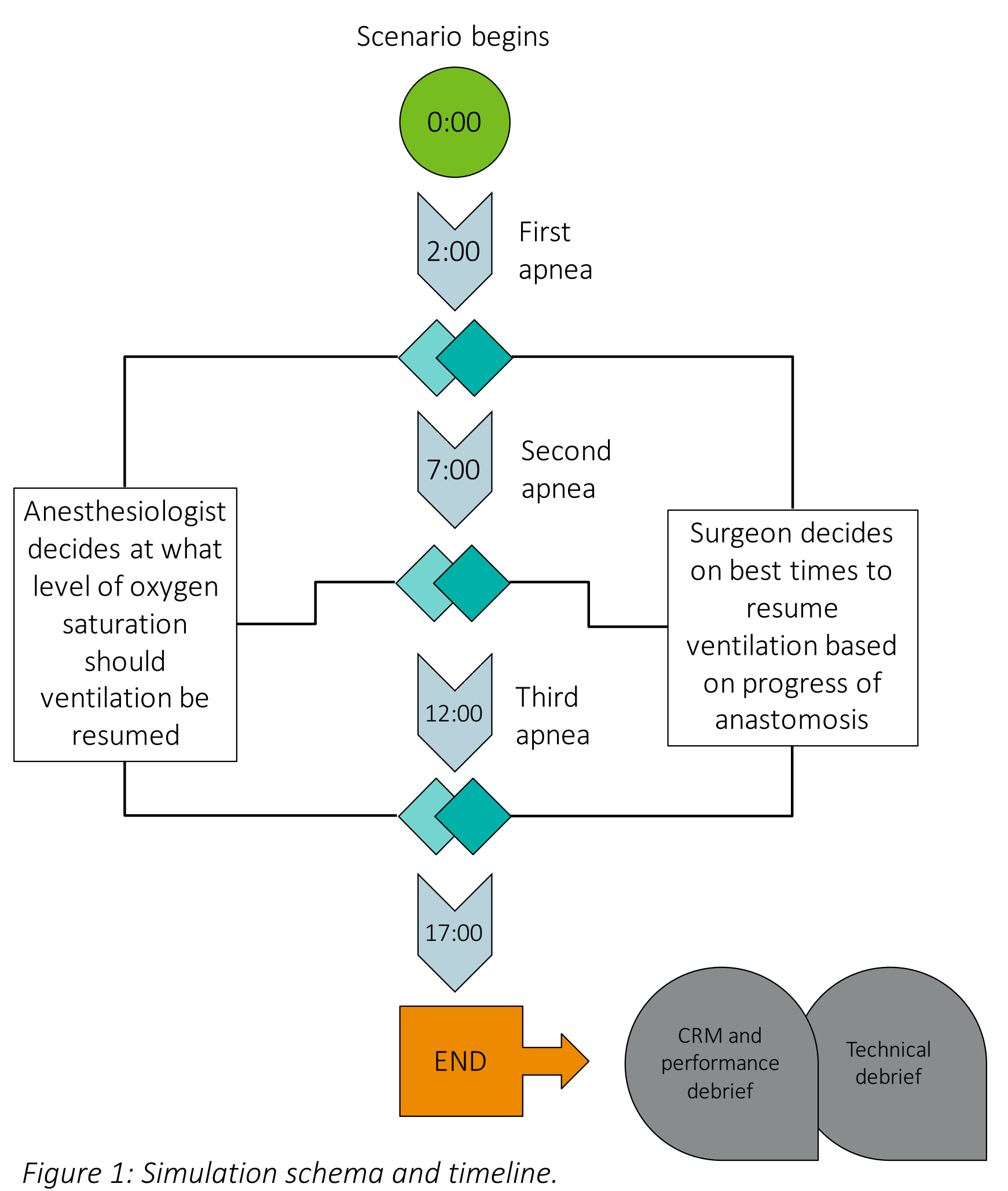

Figure 3: Surgical model installed on the Laedal SimMan. Inset shows the overall room setup including anesthesia machine and monitors.

\section{Conclusions}

This scenario highlights the utility of high-fidelity simulation for practice of non-technical crisis resource management skills, and confirms the feasibility of the scenario. Future studies will assess the effect of specific task learning and examine how this translates into the real clinical environment. 